\title{
Optical extinction for determining the size distribution of gold nanoparticles fabricated by ultra-short pulsed laser ablation
}

\author{
G.A. Torchia - L.B. Scaffardi · C. Méndez • P. Moreno • \\ J.O. Tocho $\cdot$ L. Roso
}

Received: 12 October 2007 / Accepted: 9 April 2008 / Published online: 4 July 2008

(C) Springer-Verlag 2008

\begin{abstract}
This work presents a method, based on measurements of the optical extinction spectra, to determine the size of spherical gold nanoparticles produced using the femtosecond laser ablation process in deionized water. By using an improved theoretical model that modifies the contribution of the free electrons to the dielectric function introducing a size-dependent term, it is possible to fit the full experimental extinction spectrum considering a certain size distribution. Additionally, in order to obtain complementary measurements of the size distribution, TEM analysis was performed. The results obtained showed that the predominant nanoparticle size distribution ranges from 1 to $11 \mathrm{~nm}$ in terms of radii. An optical extinction measurement together with an appropriate theoretical model based on Mie's theory represents a simple, low-cost, fast and easy method to describe a multimodal size distribution of spherical gold nanoparticles.
\end{abstract}

PACS $78.67 \mathrm{Bf} \cdot 79.20 \mathrm{Ds} \cdot 52.38 \mathrm{Ph} \cdot 06.60 \mathrm{Jn}$

G.A. Torchia ( $\varangle)$ - L.B. Scaffardi · J.O. Tocho Centro de Investigaciones Ópticas (CIOp), Casilla de Correo 124, 1900 La Plata, Argentina

e-mail: gustavot@ciop.unlp.edu.ar

G.A. Torchia · C. Méndez · P. Moreno - L. Roso Servicio Láser, Universidad de Salamanca, Plaza de la Merced s/n, 37008 Salamanca, Spain

G.A. Torchia

e-mail: gtorchia@usal.es

L.B. Scaffardi

Departamento de Ciencias Básicas, Facultad de Ingeniería, Universidad Nacional La Plata, La Plata, Argentina

J.O. Tocho

Departamento de Física, Facultad de Ciencias Exactas, Universidad Nacional de La Plata, La Plata, Argentina

\section{Introduction}

Nowadays, the generation and manipulation of nanoparticles (NPs) is a relevant topic in applied physics, required from different research areas such as biology, medicine, photonics etc. Traditionally, chemical methods are the main way to fabricate NPs which require special laboratory conditions and are not environmentally clean. In order to avoid coalescence of the NPs, this kind of production needs stabilizer or encapsulation elements, which are not adequate for biological applications or physics studies requiring pure nanomaterials.

Laser ablation represents an alternative clean method, which has been efficiently used during the previous years to produce small metal particles with size control [1,2]. As it is expected, this method constitutes a simple way to produce NPs for potential biological or medical applications, preserving the environment. Nanosecond pulse laser ablation produces large radius NPs due to the heat transferred to the bulk [3, 4]. In contrast, ultra-short pulse laser ablation produces small NPs since the laser pulse pulls off the electrons by non-linear absorption with no remarkable thermal or mechanical effects in the bulk material $[5,6]$. In fact, $\mathrm{Au}, \mathrm{Ag}$ and other metal NPs have been obtained by means of femtosecond (fs) pulse laser ablation on plane targets $[7,8]$. These works show the dependence of the particle size on the fluence and wavelength used to perform the ablation process. From these papers, it is possible to find the transition between thermal and non-thermal ablation mechanism from the shape and size distribution of the NPs. It is well known that 1-2 nm gold NPs can be obtained by fs laser ablation when surfactants are added to the surrounding medium [5]. However, these agents cannot be used to produce particles for biological applications. Recently, the first 
size control of Au NPs in pure deionized water solution was reported [9].

The optical properties of gold NPs in water solution are well known. Their extinction spectrum shows a strong absorption band centered around $520 \mathrm{~nm}$ for particles below $10 \mathrm{~nm}$ radii, and a valley centered around $450 \mathrm{~nm}$. The contrast between maximum and minimum absorption in the region from 520 to $450 \mathrm{~nm}$, respectively (contrast $=\frac{I_{520}-I_{450}}{I_{520}}$, as defined in [10]), can be used to control the size of small monomodal particles by using optical extinction spectroscopy.

As was reported previously [9], the laser ablation fabrication process can yield a broad gold NP size distribution, which depends strongly on experimental conditions. In such case, a full optical extinction spectrum fitting seems to be the appropriate procedure for sizing small NPs distribution.

In the present work, we fit the full experimental optical extinction spectrum corresponding to gold NPs fabricated by fs laser ablation in deionized water by means of the calculated extinction curve based on Mie's theory [11] obtained for a set of several monomodal size distributions of gold NPs. Additionally, the TEM technique has been conducted as an independent method for determining the NP size distribution.

\section{Experimental procedure}

The target samples of gold used to carry out these experiments were ribbons of about $500 \mathrm{~nm}$ thickness and 99.97 pure grade. Laser ablation was done using a Ti:Sapphire chirped pulsed amplification (CPA) system from Spectra Physics, emitting pulses of $120 \mathrm{fs}$ width at $1 \mathrm{kHz}$ repetition rate centered at $796 \mathrm{~nm}$ wavelength. The maximum output energy was $1.1 \mathrm{~mJ}$ per pulse with a beam waist of around $8.5 \mathrm{~mm}$. To control the incident energy, a set of neutral density filters were used. Since optical focusing is required to have a well stabilized fluence at the surface of the ribbon, a lens of $50 \mathrm{~mm}$ focal length was used. The pulse energy used provides $1000 \mathrm{~J} / \mathrm{cm}^{2}$ of fluence after focalization in air. The experimental set-up used to fabricate gold NPs is similar to that used in [12]. Under these experimental conditions we expect to find small NPs sized from 2 to $60 \mathrm{~nm}$ radii in the solution [9]. To produce the NPs the sample was moved along lines at $50 \mu \mathrm{m} / \mathrm{s}$, writing different ablation tracks in the whole surface of the sample, avoiding rewriting along the lines. The fabrication process was done during 20 minutes, after which the water solution shows a typical reddish color, which is attributed to the presence of a large number of NPs in the solution. Optical extinction spectroscopy was conducted by means of a Shimadzu spectrophotometer from 350 to $900 \mathrm{~nm}$. The sample preparation for this method precludes the development of agglomerates since no ligand compounds were added to the solutions. Besides, diluted and sonicated NP solutions were used. Complementary to this, in order to explore the size and morphology of the gold colloidal solution, TEM was performed by means of a Zeiss EM900 system.

\section{Theoretical framework}

Optical properties of materials are described by the real and the imaginary parts of the complex dielectric functions, $\varepsilon=$ $\varepsilon^{\prime}+i \varepsilon^{\prime \prime}$. For real metals, the dielectric function for the bulk can be decomposed into two terms, a free-electron term and an interband or bound-electron term [11]. Particularly, the free-electron term can be expressed by

$\varepsilon_{\text {free-electron }}(\omega)=1-\frac{\omega_{p}^{2}}{\omega^{2}+i \omega \gamma_{\text {bulk }}}$,

where $\omega_{p}$ is the plasma frequency and $\gamma_{\text {bulk }}$ is the damping constant in the Drude model.

For NPs, the electromagnetic resonances due to collective oscillations of the conduction electrons (plasmons) tend to fall in the visible region. For the case of colloidal gold, the plasmon characteristics can be used for particle sizing. As the size decreases from about 80 to $10 \mathrm{~nm}$, the plasmon peak shifts to the blue and its width narrows. However, below $10 \mathrm{~nm}$, the plasmon peak remains fixed at about $520 \mathrm{~nm}$, although the contrast between maximum and minimum $(450 \mathrm{~nm})$ varies with size. This behavior can be reproduced introducing in Mie's theory a suitable modification of the dielectric function. For particles smaller than the mean free path of conduction electrons in the bulk metal, the mean free path becomes dominated by collisions with the particle boundary [13]. The damping constant (inverse of the collision time for conduction electrons in the Drude theory) is increased due to these additional collisions, and it can be written as

$\gamma_{\text {size }}(r)=\gamma_{\text {bulk }}+K \frac{v_{F}}{r}$,

where $v_{F}$ is the electron velocity at the Fermi surface and $r$ is the radius of the nanoparticle. Taking into account (2) and introducing it in (1), it is possible to calculate the extinction spectra for gold NPs [10]. Values of $v_{F}=14.1 \times 10^{14} \mathrm{~nm} / \mathrm{s}$, $\omega_{p}=1.3 \times 10^{16} \mathrm{~s}^{-1}$ and $\gamma_{\text {bulk }}=1.64 \times 10^{14} \mathrm{~s}^{-1}$ were taken from [14]. The value of $K$ (dimensionless) was determined by fitting several experimental extinction spectra corresponding to standard gold nanoparticles. The best fit was obtained for $K=1$ [10].

The extinction cross section for small spheres compared with the incident wavelength can be written as [11]

$C_{\mathrm{ext}}=\frac{2 \pi}{\lambda} \operatorname{Im}\{\alpha\}=\pi r^{2} x \operatorname{Im}\left\{\frac{\varepsilon_{1}-\varepsilon_{m}}{\varepsilon_{1}+2 \varepsilon_{m}}\right\}$ 

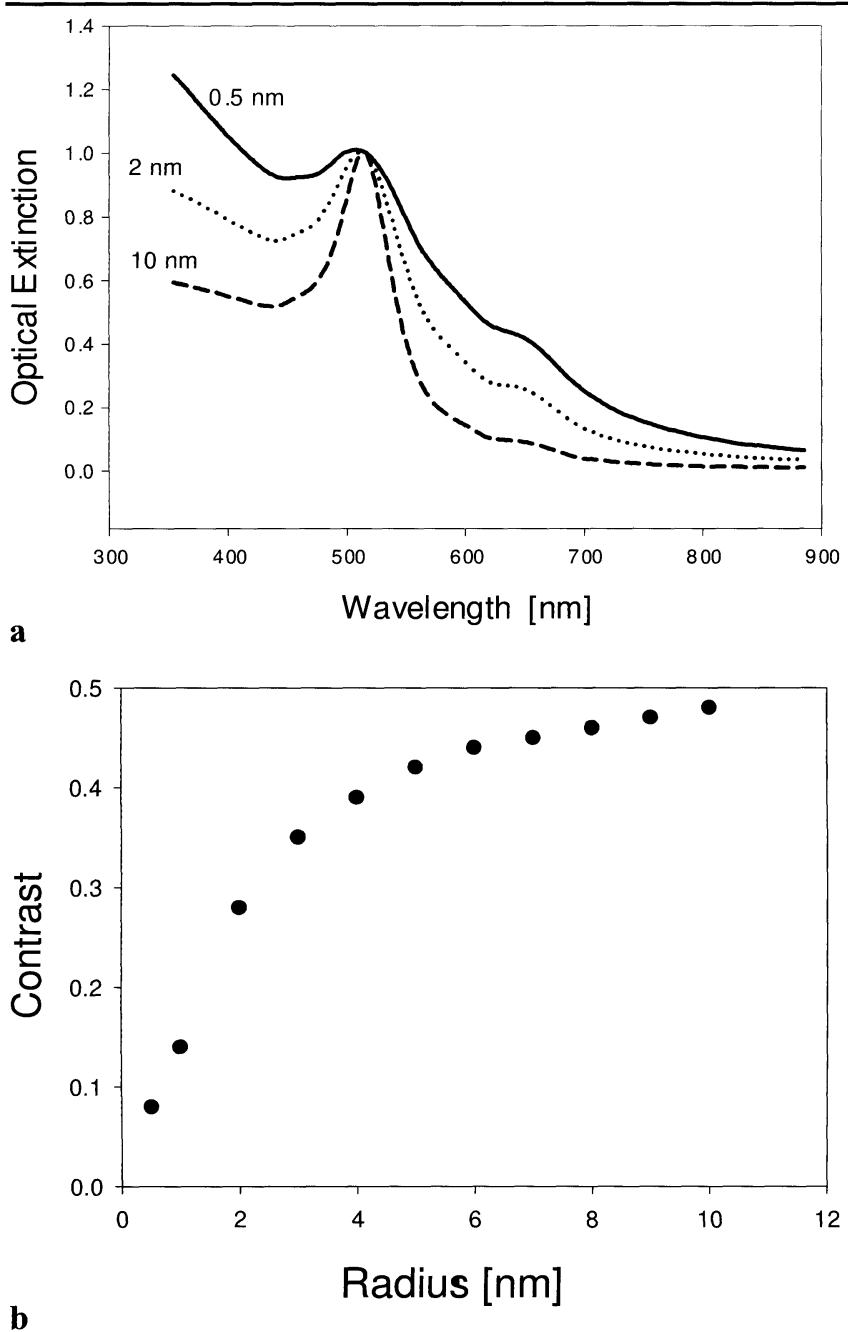

Fig. 1 (a) Calculated optical extinction spectra corresponding to gold NPs of $0.5,2$ and $10 \mathrm{~nm}$; (b) contrast as a function of the different NPs radii

where $\lambda$ is the incident wavelength, $\alpha$ is the polarizability, $r$ is the particle radius, $\varepsilon_{1}$ is the dielectric function of the particle, $\varepsilon_{m}$ is the dielectric function of the medium and $x=\frac{2 \pi r}{\lambda} n_{m}$ is the size parameter, with $n_{m}$ being the medium refractive index.

Figure 1a shows calculated optical extinction spectra for different sizes, and Fig. $1 \mathrm{~b}$ the contrast as a function of the radius of the gold NPs. To fit the experimental optical extinction spectra, it is necessary to consider a theoretical extinction cross section as a linear combination of monomodal contributions of different sizes:

$C_{\mathrm{ext}}=\sum_{i} k_{i} C_{i}$

where $C_{i}$ is the extinction cross section (in $\mathrm{cm}^{2}$ ) for each monomodal size of radius $r_{i}$ and $k_{i}=\frac{N_{i}}{N}$ is defined as the ratio between the number of particles of size $r_{i}$ and the total number $N$ per unit volume.

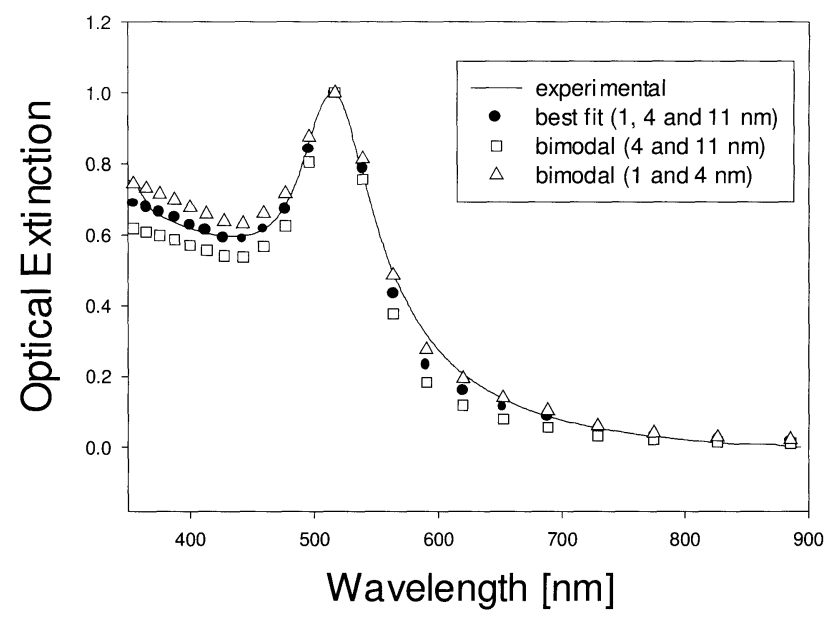

Fig. 2 The solid line represents the experimental results. Full circles correspond to the best fit including 1, 4 and $11 \mathrm{~nm}$. Triangles and squares correspond to different bimodal distributions

\section{Results and discussion}

The experimental optical extinction spectrum for a colloidal gold NP solution was obtained for the conditions mentioned before. The sample used for optical measurements was carefully prepared with a low NP concentration in order to assure the single scattering regime. The experimental data are displayed in Fig. 2 (solid line). As can be seen, the strong resonance plasmon is centered at $520 \mathrm{~nm}$ in accordance with the values reported in previous works [11]. This peak corresponds to the optical extinction for gold NPs in deionized water solution whose sizes range below $10 \mathrm{~nm}$ radii [8]. On the short-wavelength side of the resonance peak, a valley centered at $450 \mathrm{~nm}$ can be observed. In a previous work [10] it has been observed that while the valley position is centered at $450 \mathrm{~nm}$, the value of the optical extinction at this point varies as a function of the radius, suggesting that the contrast may be used for sizing particles below $5 \mathrm{~nm}$.

From our experimental results, a contrast value of 0.40 was obtained. From Fig. 1b, this allows for a rough characterization of the particle size distribution around $4 \mathrm{~nm}$.

The preliminary size value suggested by the analysis of the contrast may be interpreted as an average particle size obtained for the gold NP solution. In order to obtain complete information about the particle distribution in the sample, we analyzed the full optical extinction spectrum.

In this sense, to fit the experimental data (solid line), we have calculated the optical extinction curve considering a distribution of several monomodal size particles. The results in Fig. 2 (full circles) represent the best fit to the experimental data using a calculated curve which includes three sizes: $1 \mathrm{~nm}(92 \%), 4 \mathrm{~nm}(7.8 \%)$ and $11 \mathrm{~nm}(0.2 \%)$. Additionally, we also show the results for two other bimodal distributions: $1 \mathrm{~nm}(92 \%)$ and $4 \mathrm{~nm}(8 \%)$ particles (triangles) and $4 \mathrm{~nm}$ 


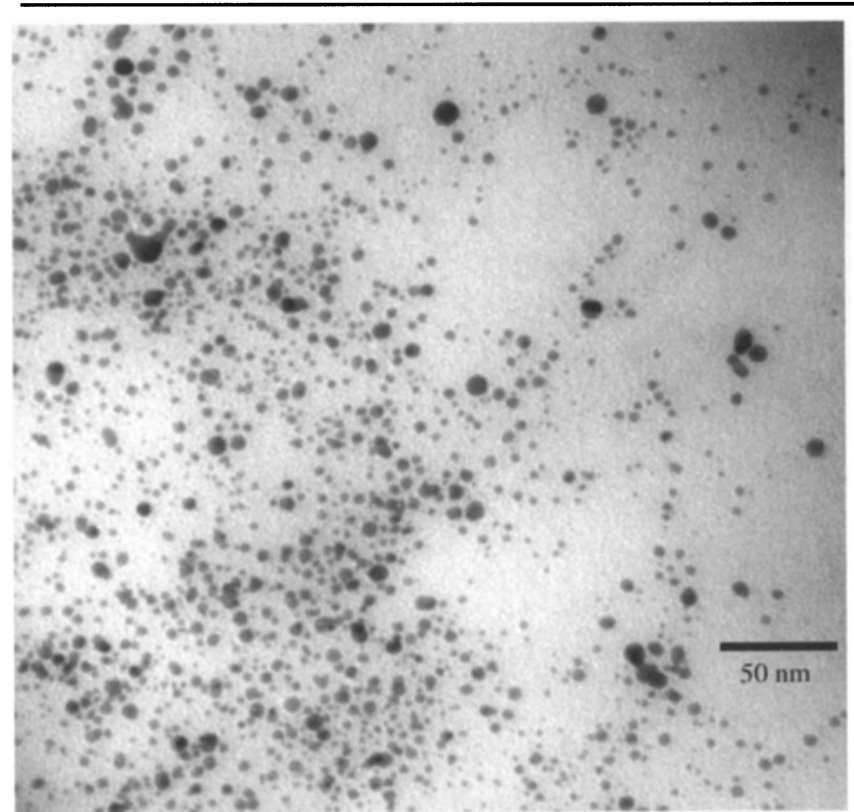

a

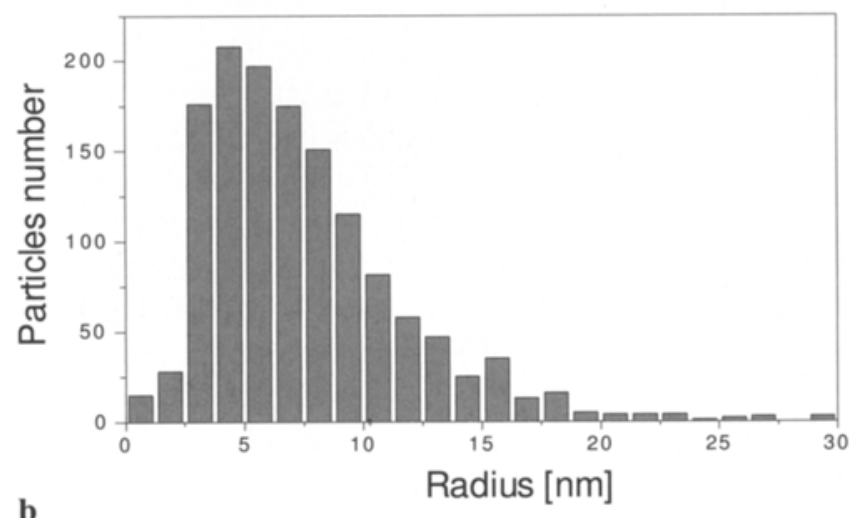

Fig. 3 (a) TEM picture of NPs in water solution made by fs laser ablation; (b) histogram corresponding to the size distribution of gold nanoparticles fabricated by fs laser ablation with high fluence

$(90 \%)$ and $11 \mathrm{~nm}(10 \%)$ (squares). The latter were included to show the influence of the addition of particles smaller (triangles) and larger (squares) than the average on the shape of the extinction spectrum. As can be seen from Fig. 2, these bimodal distributions cannot fit the experimental curve.

Finally, in order to obtain an additional characterization of the analyzed gold particles solution, we have performed TEM. The preparation of the TEM samples was done by using a polymer copper microgrid. The samples were carefully dried in an oven slightly above room temperature. TEM size measurements do not have enough resolution to observe the smallest particles, and errors resulting from sampling can be quite important. Since the particles were not homogeneously distributed inside the grid, conclusions about the size distribution may not be the most appropriate, although the areas under observation were chosen randomly.
Figure 3a shows a typical TEM picture of gold NPs fabricated by means of ultra-short laser ablation in solution. As can be seen, there is a large number of small particles $(<10 \mathrm{~nm})$. In many TEM pictures, agglomerated gold NPs were also observed. This effect is a consequence of the TEM sample preparation. There are also a small number of large particles $(>25 \mathrm{~nm})$. In order to measure the size and the number of the particles present in the gold sample, we have developed software to identify and measure the NPs size from the TEM picture, disregarding the agglomerates. The number of particles counted by the software is above one thousand, which guarantees an acceptable statistics for the NP size distribution.

From this procedure we have obtained the size and number of the gold NP distribution (Fig. 3b). The histogram was constructed exploring many TEM pictures corresponding to the same sample.

The histogram shows a large number of NPs centered at $4 \mathrm{~nm}$ with an additional contribution centered around $12 \mathrm{~nm}$, which is in accordance with results reported by other authors [8]. A careful observation shows a small contribution of particles below $2 \mathrm{~nm}$. Since these are close to the resolution limit of the TEM $(1 \mathrm{~nm})$, the results may not be reliable in this range. Thus, it must be remarked that the smaller particles included in the theoretical curve used to fit the experimental extinction spectrum (Fig. 2) have not been identified in the TEM measurements.

The production of NPs with largest sizes is related to phase explosion thermal processes induced in the gold target by means of ultra-short laser ablation.

The contribution of the three sizes $(1,4$ and $11 \mathrm{~nm})$ used to fit the experimental extinction spectrum has a finite width centered on those values. However, this consideration does not affect the final fit. This statement is supported by Fig. 3b where a non-discrete NP size distribution shows to be a more realistic assumption.

\section{Conclusions}

In summary, we have presented an effective optical extinction method to determine a mix of several monomodal size distributions of gold NPs in water solution obtained by fs laser ablation with a high fluence. The main sizes obtained after the optical extinction analysis were $1 \mathrm{~nm}(92 \%), 4 \mathrm{~nm}$ $(7.8 \%)$ and $11 \mathrm{~nm}(0.2 \%)$. The results of TEM microscopy agree reasonably well with the optical spectroscopy measurements, taking into account the limited resolution $(1 \mathrm{~nm})$ for our TEM system.

We have shown that the optical extinction method is extremely sensitive for distinguishing slightly different size contributions for fitting the experimental spectra. In this sense, it allows for measurements of small particles which 
are beyond the TEM resolution limit. The optical extinction method represents a simple, fast, low-cost and reliable technique for sizing gold NPs independently of the fabrication method, complementary to TEM analysis.

Acknowledgements This work has been partially supported by the Ministerio de Ciencia y Tecnología (FIS2006-04151 MEC, Spain), by the Agencia Española de Cooperación Internacional (AECI, Spain) under project A/3652/05, by CONICET (PIP 5997) and by the Agencia Nacional de Promoción Científica y Tecnológica (PICT 15210 and PICT 26090) from Argentina.

\section{References}

1. A. Henglein, J. Phys. Chem. 97, 5457 (1993)

2. M.S. Sibbald, G. Chumanov, T.M. Cotton, J. Phys. Chem. 100, 4672 (1996)

3. M.S. Yeh, Y.S. Yang, Y.P. Lee, H.F. Lee, Y.H. Yeh, C.S. Yeh, J. Phys. Chem. B 103, 6851 (1999)
4. F. Mafune, J. Kohno, Y. Takeda, T. Kondow, H. Sawabe, J. Phys. Chem. B 104, 8333 (2000)

5. A.V. Kabashin, M. Meunier, C. Kingston, J.T. Luong, J. Phys. Chem. B 107, 4527 (2003)

6. T. Tsuji, K. Iryo, N. Watanabe, M. Tsuji, Appl. Surf. Sci. 202, 80 (2002)

7. T. Tsuji, T. Kakita, M. Tsuji, Appl. Surf. Sci. 206, 314 (2003)

8. S. Barcikowski, A. Hahn, A.V. Kabashin, B.N. Chichkov, Appl. Phys. A 87, 47-55 (2007)

9. A.V. Kabashin, M. Meunier, J. Appl. Phys. 94, 7941 (2003)

10. L.B. Scaffardi, N. Pellegri, O. de Sanctis, J.O. Tocho, Nanotechnology 16, 158-163 (2005)

11. C.F. Bohren, D.R. Huffman, Absorption and Scattering of Light by Small Particles (Wiley, New York, 1998)

12. P. Moreno, C. Méndez, A. García, G.A. Torchia, D. Delgado, J.R. Vázquez de Aldana, I. Arias, L. Roso, J. Nanosci. Nanotechnol. 6, 1961-1967 (2006)

13. U. Kreibig, C. von Fragstein, Z. Phys. 224, 307-323 (1969)

14. C.G. Granqvist, O. Hunderi, Phys. Rev. B 16, 3513-3534 (1977) 